\title{
Use of Polynomial Shape Function in Shear Deformation Theory for Thick Plate Analysis
}

\author{
${ }^{1}$ Ibearugbulem, Owus M., ${ }^{2}$ Gwarah, \\ Ledum S., ${ }^{3}$ Ibearugbulem, C. N. \\ ${ }^{1,2,3}$ Civil Engineering Department, Federal University of Technology, Owerri, Nigeria
}

\begin{abstract}
This paper presents use of polynomial shape function in shear deformation theory for thick plate analysis. Total potential energy equation of a thick plate was formulated from the first principle. This equation was subjected to direct variation to obtain three simultaneous direct governing equations for determination of displacement coefficients. Shape (profile) equation for vertical shear stress through the thickness of the plate was formulated from the first principle. From this profile equation, the deformation line equation (called function of $\mathrm{z}$ or s) was obtained. This is the model from this study. A numerical problem for a rectangular plate simply supported around all the edges was used to test the sufficiency of this study. Both polynomial and trigonometric shape functions were used in this problem. Three other models were also used. The center deflection - $\mathrm{w}(0.5,0.5,0)$, in-plane normal $\mathrm{x}$ directed stress $\sigma_{\mathrm{x}}(0.5,0.5,0.5)$ and $\mathrm{x}$ directed vertical shear stress $\tau_{x z}(0,0.5,0)$ from the present study for span-depth ratio of 4 of a square plate using polynomial shape function are $0.0055 \mathrm{qa}^{4} / \mathrm{D}, 0.03 \mathrm{tqa}^{2} / \mathrm{D}$ and $0.00149 \mathrm{qa}^{3} / \mathrm{D}$. D is plate flexural rigidity, $\mathrm{t}$ is plate thickness, $\mathrm{q}$ is the uniformly distributed normal load on plate and a is the primary span of the plate. When Trigonometric shape function is used, the values are $0.0056 \mathrm{qa}^{4} / \mathrm{D}, 0.031 \mathrm{tqa}^{2} / \mathrm{D}$ and $0.00147 \mathrm{qa}^{3} / \mathrm{D}$. These are comparable with the value from Pagano (Exact): $0.0052 \mathrm{qa}^{4} / \mathrm{D}, 0.0281 \mathrm{tqa}^{2} / \mathrm{D}$ and $0.00117 \mathrm{qa}^{3} / \mathrm{D}$. It is observed that at span-depth ratio of up to 100 the values based on all the models used herein coincide with the Classical plate theory (CPT) values. The values of from CPT based on polynomial shape function are $0.00414 \mathrm{qa}^{4} / \mathrm{D}, 0.0283 \mathrm{tqa}^{2} / \mathrm{D}$ and 0.00 $\mathrm{qa}^{3} / \mathrm{D}$.
\end{abstract}

Keywords: shear deformation, vertical shear stress, stress, deflection, displacement, potential energy shape function

\section{INTRODUCTION}

Refined plate theories have been characterized by the use of trigonometric displacement function. Many scholars have obtained the closed form solutions and others have obtained approximate solution by use of energy method. However, one thing is common in them all - the use of trigonometric displacement functions to approximate the deformed shapes of the plates. (Chikalthankar et al., 2013; Sayyad, 2011; Akavci, 2007; Sayyad and Ghugal, 2012; Sadrnejad et al., 2009; Daouadji et al.,2013;Hashemi and Arsanjani, 2005; Reddy, 2014; Shimpi and Patel, 2006; Murthy, 1981; Daouadji, Tounsi, Hadji, Henni and El Abbes, 2012; Zhen-qiang, Xiu:xi and Mao-guang, 1994). Others have applied the polynomial displacement functions in numerical methods like finite element method and differential quadrature element methods (Matikainen, Schwab and Mikkola, 2009; Goswami and Becker, 2013, Liu, 2001). In the course of development of refined plate theory, the assumption that the shear deformation line is not varying linear with depth of the plate was introduced. This according to many scholars helps to ensure that the vertical shear stress across the plate section does not remain constant, but varies parabolically with zero values at both the top and bottom surfaces (Kruszewski, 1949; Ambartsumian, 1958 Krishna, 1984; Touratier, 1991; Karama and Mistou, 2003; Sayyad, 2011). They came up with different shear deformation line functions, here-in-after called $F(z)$. However, there $F(z)$ were not strictly based on the vertical shear stress mathematical formulation. If we follow the work of Timoshenko (Timoshenko and Woinowsky-krieger, 1970), we shall note that maximum shear stress occurs at the mid surface (where $\mathrm{z}=0$ ) and the value of maximum shear stress is one and half of vertical shear stress. With most of the $F(z)$ from the literature, we may obtain good profile (curve) for the deformation line and shear stress distribution across the section, but the mid surface value of shear stress may not coincide with that from Timoshenko. Thus, the two specific objectives of the present study include

i. To develop a direct governing simultaneous equations for thick plate analysis

ii. To formulation of a polynomial F(z) mathematical in line with works of Timoshenko

iii. To use polynomial displacement functions variational calculus analysis. 


\section{ASSUMPTIONS}

i. The displacements, $\mathrm{u}, \mathrm{v}$ and $\mathrm{w}$ are small when compared with plate thickness.

ii. The in-plane displacements, $\mathrm{u}$ and $\mathrm{v}$ are differentiable in $\mathrm{x}, \mathrm{y}$ and $\mathrm{z}$ axes, while the out-of-plane displacement (deflection), $\mathrm{w}$ is only differentiable in $\mathrm{x}$ and $\mathrm{y}$ axes. This means that the first derivative of $\mathrm{w}$ with respect to $\mathrm{z}$ is zero. Consequently, $\varepsilon_{\mathrm{z}}=0$.

iii. The effect of the out-of-plane normal stress on the gross response of the plate is small when compared with other stresses. Thus, it can be neglected. That is $\sigma_{z}=0$.

iv. The vertical line that is initially normal to the middle surface of the plate before bending is no longer straight nor normal to the middle surface after bending. That is $\quad \phi \neq \theta_{\mathrm{c}}$. where $\phi$ is the total rotation of the middle surface in this case, $\theta_{c}$ is the classical plate theorem rotation of the middle surface.

\section{KINEMATIC RELATIONS}

The refined plate theory (RPT) in-plane displacements, $\mathrm{u}$ and $\mathrm{v}$ as presented on figure lare defined mathematically as

$$
\begin{aligned}
& u=u_{c}+u_{s} \\
& v=v_{c}+v_{s}
\end{aligned}
$$

Where $\mathrm{u}$ and $\mathrm{v}$ are in-plane displacements in $\mathrm{x}$ and $\mathrm{y}$ directions respectively.

The classical in-plane displacements are commonly defined as:

$$
\begin{aligned}
& u_{c}=-z \theta_{c x}=z \frac{d w}{d x} \\
& v_{c}=-z \theta_{c y}=z \frac{d w}{d y}
\end{aligned}
$$

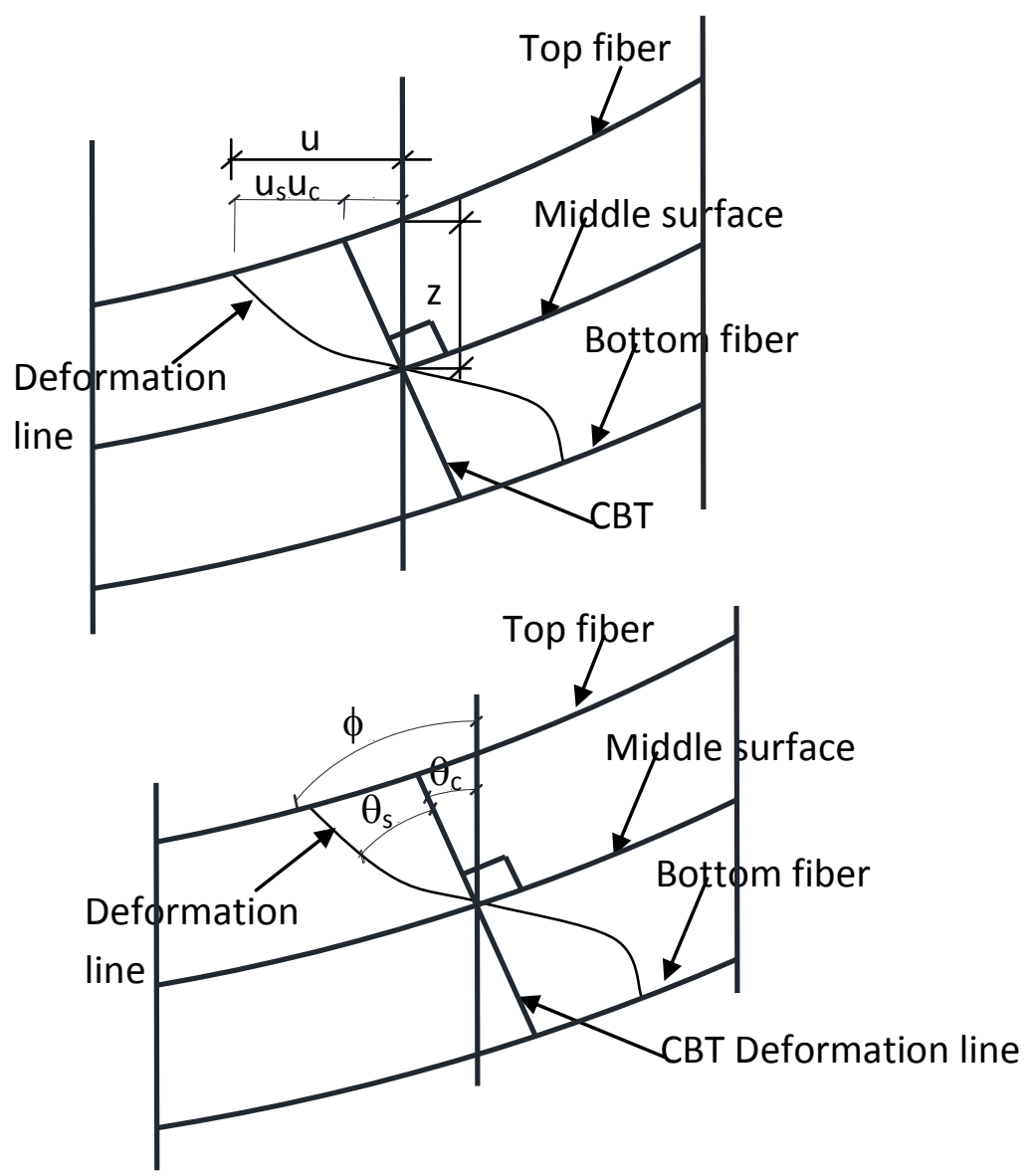

Figure 1: Deformation of a section of a thick plate 
Analogously, the shear deformation components of the in-plane displacements are defined as:

$$
\begin{aligned}
& u_{s}=F(z) \theta_{s x} \\
& v_{s}=F(z) \theta_{s y}
\end{aligned}
$$

$\operatorname{NoteF}(z)$ is used instead of $z$. This is because of the fourth assumption (see figure 1). Let us define the deflection (out-of-plane displacement), w as:

$w=c_{1} h$

(7)

Substituting equation (7) into equations (3) and (4) gives respectively:

$$
\begin{aligned}
& u_{c}=-z \frac{d}{d x}\left(c_{1} h\right)=-z c_{1} \frac{d h}{d x} \\
& v_{c}=-z \frac{d}{d y}\left(c_{1} h\right)=-z c_{1} \frac{d h}{d y}
\end{aligned}
$$

Let us mimic expressions in equations (8) and (9) rewrite equations (5) and (6) as:

$$
\begin{aligned}
& u_{s}=F(z) c_{2} \frac{d h}{d x} \\
& v_{s}=F(z) c_{3} \frac{d h}{d y}
\end{aligned}
$$

Where $c_{1}, c_{2}$ and $c_{3}$ are coefficients of deflection (w), shear deformation rotations $\left(\theta_{\mathrm{sx}}\right.$ and $\left.\theta_{\mathrm{sy}}\right)$.

Substituting equations (3) and (10) into equation (1) gives:

$$
u=\left(-c_{1} z+c_{2} F(z)\right) \frac{d h}{d x}
$$

Similarly, substituting equations (4) and (11) into equation (2) gives:

\section{Strain - Displacement Relations}

$$
v=\left(-c_{1} z+c_{3} F(z)\right) \frac{d h}{d y}
$$

It was assumed that $\varepsilon_{\mathrm{z}}$ is equal to zero. Thus, the remaining five engineering strain components are defined as:

$$
\begin{aligned}
\varepsilon_{x} & =\frac{d u}{d x}=\left[-c_{1} z+c_{2} F(z)\right] \frac{d^{2} h}{d x^{2}} \quad(14) \\
\varepsilon_{y} & =\frac{d v}{d y}=\left[-c_{1} z+c_{3} F(z)\right] \frac{d^{2} h}{d y^{2}} \quad \text { (15) } \\
\gamma_{x y}=\frac{d u}{d y}+\frac{d v}{d x} & =\left[-c_{1} z+c_{2} F(z)\right] \frac{d^{2} h}{d x d y}+\left[-c_{1} z+c_{3} F(z)\right] \frac{d^{2} h}{d x d y} \\
=\left[-2 c_{1} z+c_{2} F(z)+c_{3} F(z)\right] \frac{d^{2} h}{d x d y} \quad(16) & \gamma_{x z}=\frac{d u}{d z}+\frac{d w}{d x} \\
& =\left[-c_{1}+c_{2} \frac{d F(z)}{d z}\right] \frac{d h}{d x}+c_{1} \frac{d h}{d x} \\
\gamma_{x z} & =c_{2} \frac{d F(z)}{d z} \frac{d h}{d x} \\
& =c_{3} \frac{d F(z)}{d z} \frac{d h}{d y}=\frac{d v}{d z}+\frac{d w}{d y} \\
& =\left[\begin{array}{c}
\text { That is } \\
\left.\gamma_{1}+c_{3} \frac{d F(z)}{d z}\right] \frac{d h}{d y}+c_{1} \frac{d h}{d y}
\end{array}\right.
\end{aligned}
$$

\section{CONSTITUTIVE RELATIONS}

It was assumed that $\sigma_{\mathrm{z}}$ is equal to zero. Thus, the remaining five stress components are defined as: 


$$
\begin{aligned}
& \sigma_{x}=\frac{E}{1-\mu^{2}}\left[\varepsilon_{x}+\mu \varepsilon_{y}\right] \\
& \sigma_{y}=\frac{E}{1-\mu^{2}}\left[\mu \varepsilon_{x}+\varepsilon_{y}\right] \\
& \tau_{x y}=\frac{E(1-\mu)}{1-\mu^{2}} \gamma_{x y} \\
& \tau_{x z}=\frac{E(1-\mu)}{1-\mu^{2}} \gamma_{x z} \\
& \tau_{y z}=\frac{E(1-\mu)}{1-\mu^{2}} \gamma_{y z}
\end{aligned}
$$

\section{STRESS - DISPLACEMENT EQUATIONS}

Substituting equations (14) to (18) into equations (19) to (23) where appropriate gives:

$$
\begin{gathered}
\sigma_{x}=\frac{E}{1-\mu^{2}}\left[\left[-c_{1} z+c_{2} F(z)\right] \frac{d^{2} h}{d x^{2}}+\mu\left[-c_{1} z+c_{3} F(z)\right] \frac{d^{2} h}{d y^{2}}\right] \\
\sigma_{y}=\frac{E}{1-\mu^{2}}\left[\mu z\left(c_{1}+\frac{F(z)}{z} B_{2}\right) \frac{d^{2} h}{d x^{2}}+\left[-c_{1} z+c_{3} F(z)\right] \frac{d^{2} h}{d y^{2}}\right] \\
\tau_{x y}=\frac{E(1-\mu)}{2\left(1-\mu^{2}\right)}\left[-2 c_{1} z+c_{2} F(z)+c_{3} F(z)\right] \frac{d^{2} h}{d x d y} \\
\tau_{x z}=\frac{E(1-\mu)}{2\left(1-\mu^{2}\right)} c_{2} \frac{d F(z)}{d z} \frac{d h}{d x} \\
\tau_{y z}=\frac{E(1-\mu)}{2\left(1-\mu^{2}\right)} c_{3} \frac{d F(z)}{d z} \frac{d h}{d y}
\end{gathered}
$$

\section{TOTAL POTENTIAL ENERGY}

Total potential energy is the summation of strain energy, $\mathrm{U}$ and external work, V. that's

$$
\Pi=U+V
$$

Let's define external work as:

$$
V=-q \int_{x} \int_{y} w d x d y
$$

Let's also define strain energy mathematically:

$$
\begin{gathered}
U=\int_{x} \int_{y}\left[\int_{-\frac{t}{2}}^{\frac{t}{2}} \sigma \cdot \varepsilon d z\right] d x d y \\
=\int_{x} \int_{y}\left[\int_{-\frac{t}{2}}^{\frac{t}{2}}\left(\sigma_{x} \varepsilon_{x}+\sigma_{y} \varepsilon_{y}+\tau_{x y} \gamma_{x y}+\tau_{x z} \gamma_{x z}+\tau_{y z} \gamma_{y z}\right) d z\right] d x d y
\end{gathered}
$$

Using equations (14) and (24), (15) and (25), (16) and (26), (17) and (27), and (18) and (28) respectively gives:

$$
\begin{gathered}
\sigma_{x} \varepsilon_{x}=\frac{E}{1-\mu^{2}}\left[\left[z^{2} c_{1}{ }^{2}-2 c_{1} c_{2} z F(z)+c_{2}{ }^{2} F(z)^{2}\right]\left(\frac{d^{2} h}{d x^{2}}\right)^{2}\right. \\
\left.+\mu\left[z^{2} c_{1}{ }^{2}-c_{1} c_{2} z F(z)-c_{1} c_{3} z F(z)+c_{2} c_{3} F(z)^{2}\right]\left(\frac{d^{2} h}{d x d y}\right)^{2}\right]
\end{gathered}
$$




$$
\begin{gathered}
\sigma_{y} \varepsilon_{y}=\frac{E}{1-\mu^{2}}\left[\left[z^{2} c_{1}{ }^{2}-2 c_{1} c_{3} z F(z)+c_{3}^{2} F(z)^{2}\right]\left(\frac{d^{2} h}{d y^{2}}\right)^{2}\right. \\
\left.+\mu\left[z^{2} c_{1}^{2}-c_{1} c_{2} z F(z)-c_{1} c_{3} z F(z)+c_{2} c_{3} F(z)^{2}\right]\left(\frac{d^{2} h}{d x d y}\right)^{2}\right] \\
\tau_{x y} \cdot \gamma_{x y}=\frac{E(1-\mu)}{2\left(1-\mu^{2}\right)}\left[4 c_{1}{ }^{2} z^{2}-4 c_{1} c_{2} z F(z)-4 c_{1} c_{3} z F(z)+c_{2}{ }^{2} F(z)^{2}+2 c_{2} c_{3} F(z)^{2}\right. \\
\left.+c_{3}^{2} F(z)^{2}\right]\left(\frac{d^{2} h}{d x d y}\right)^{2} \\
\tau_{x z} \cdot \gamma_{x z}=\frac{E(1-\mu)}{2\left(1-\mu^{2}\right)} c_{2}{ }^{2}\left[\frac{d F(z)}{d z}\right]^{2}\left(\frac{d h}{d x}\right)^{2} \\
\tau_{y z} \cdot \gamma_{y z}=\frac{E(1-\mu)}{\left(1-\mu^{2}\right)} c_{3}{ }^{2}\left[\frac{d F(z)}{d z}\right]^{2}\left(\frac{d h}{d y}\right)^{2}
\end{gathered}
$$

Substituting equations (32) to (36) into equation (31) gives:

$$
\begin{gathered}
U=\frac{D}{2} \int_{x} \int_{y}\left[g_{1} c_{1}{ }^{2}-2 g_{2} c_{1} c_{2}+g_{3} c_{2}{ }^{2}\right]\left(\frac{d^{2} h}{d x^{2}}\right)^{2} \\
+\left[2 g_{1} c_{1}{ }^{2}-2 g_{2} c_{1} c_{2}-2 g_{2} c_{1} c_{3}+\frac{1}{2} g_{3} c_{2}{ }^{2}+g_{3} c_{2} c_{3}+\frac{1}{2} g_{3} c_{3}{ }^{2}\right]\left(\frac{d^{2} h}{d x d y}\right)^{2} \\
+\mu\left[g_{3} c_{2} c_{3}-\frac{1}{2} g_{3} c_{2}{ }^{2}-\frac{1}{2} g_{3} c_{3}^{2}\right]\left(\frac{d^{2} h}{d x d y}\right)^{2} \\
+\left[g_{1} c_{1}{ }^{2}-2 g_{2} c_{1} c_{3}+g_{3} c_{3}^{2}\right]\left(\frac{d^{2} h}{d y^{2}}\right)^{2} \\
+(1-\mu) \frac{\alpha^{2}}{2} g_{4} c_{2}{ }^{2}\left(\frac{d h}{d x}\right)^{2} \\
\left.+(1-\mu) \frac{\alpha^{2}}{2} g_{4} B_{3}{ }^{2}\left(\frac{d h}{d y}\right)^{2}\right] d x d y
\end{gathered}
$$

Where:

$$
\begin{gathered}
\bar{D}=\frac{t^{3}}{12} \\
g_{1}=\frac{\left(\int_{-\frac{t}{2}}^{\frac{t}{2}} z^{2} d z\right)}{\bar{D}}=1 \\
g_{2}=\frac{\left(\int_{-\frac{t}{2}}^{\frac{t}{2}} z F(z) d z\right)}{\bar{D}} \\
g_{3}=\frac{\left(\int_{-\frac{t}{2}}^{\frac{t}{2}} F(z)^{2} d z\right)}{\bar{D}}
\end{gathered}
$$




$$
\alpha^{2} g_{4}=\frac{\left(\int_{-\frac{t}{2}}^{\frac{t}{2}}\left[\frac{d F(z)}{d z}\right]^{2} d z\right)}{\bar{D}}(41)
$$

The flexural rigidity of the plate is:

$$
D=\frac{E}{1-\mu^{2}} * \bar{D}=\frac{E t^{3}}{12\left(1-\mu^{2}\right)}
$$

Let's define the span-depth aspect ratio as

$$
\alpha=\frac{a}{t}
$$

Where $\mathrm{a}$ and $\mathrm{t}$ are the primary span (length in $\mathrm{x}$ direction, while $\mathrm{b}$ is the length in $\mathrm{y}$ direction) of the plate and plate thickness respectively.

Let define non dimensional coordinates $\mathrm{R}$ and $\mathrm{Q}$ and the span-span aspect ratio, $\mathrm{P}$ as:

$$
\begin{gathered}
R=\frac{x}{a} \Rightarrow x=a R \\
Q=\frac{y}{b} \Rightarrow y=b Q \\
P=\frac{b}{a} \Rightarrow b=a P
\end{gathered}
$$

Substituting equations (30), (37) and (43) to (46)into equation (29) gives:

$$
\begin{gathered}
\Pi=\frac{a b D}{2 a^{4}} \int_{0}^{1} \int_{0}^{1}\left[g_{1} c_{1}{ }^{2}-2 g_{2} c_{1} c_{2}+g_{3} c_{2}{ }^{2}\right]\left(\frac{d^{2} h}{d R^{2}}\right)^{2} \\
+\frac{1}{P^{2}}\left[2 g_{1} c_{1}^{2}-2 g_{2} c_{1} c_{2}-2 g_{2} c_{1} c_{3}\right]\left(\frac{d^{2} h}{d R d Q}\right)^{2} \\
\frac{(1+\mu)}{P^{2}} g_{3} c_{2} c_{3}\left(\frac{d^{2} h}{d R d Q}\right)^{2} \\
+\frac{(1-\mu)}{2 P^{2}}\left[g_{3} c_{2}{ }^{2}+g_{3} c_{3}{ }^{2}\right]\left(\frac{d^{2} h}{d R d Q}\right)^{2} \\
+\frac{(1-\mu) \alpha^{2}}{2} g_{4} c_{2}{ }^{2}\left(\frac{d h}{d R}\right)^{2} \\
\left.+\frac{(1-\mu) \alpha^{2}}{2 P^{2}} g_{4} c_{3}^{2}\left(\frac{d h}{d Q}\right)^{2}\right] d R d Q \\
-a b \int_{0}^{1} \int_{0}^{1} F F d R d Q
\end{gathered}
$$

\section{DIRECT GOVERNING EQUATIONS}

This total potential energy contains three unknown coefficients $\left(c_{1}, c_{2}\right.$ and $\left.c_{3}\right)$ for deflection, rotation in $\mathrm{x}$ axis and rotation in y axis. Differentiating total potential energy equation with respect to $c_{1}, c_{2}$ and $c_{3}$ in turn will give three simultaneous equations.

$$
\frac{d \Pi}{d c_{1}}=\frac{d \Pi}{d c_{2}}=\frac{d \Pi}{d c_{3}}=0
$$

Substituting equation (47) into equation (48) gives in matrix form:

$$
\begin{gathered}
{\left[\begin{array}{lll}
r_{11} & r_{12} & r_{13} \\
r_{12} & r_{22} & r_{23} \\
r_{13} & r_{23} & r_{33}
\end{array}\right]\left[\begin{array}{l}
c_{1} \\
c_{2} \\
c_{3}
\end{array}\right]=\frac{a^{4}}{D}\left[\begin{array}{c}
F_{r q} \\
0 \\
0
\end{array}\right]} \\
r_{11}=g_{1}\left(k_{1}+2 \frac{k_{2}}{P^{2}}+\frac{k_{3}}{P^{4}}\right) \\
r_{12}=-g_{2}\left(k_{1}+\frac{k_{2}}{P^{2}}\right)
\end{gathered}
$$




$$
\begin{gathered}
r_{13}=-g_{2}\left(\frac{k_{2}}{P^{2}}+\frac{k_{3}}{P^{4}}\right) \\
r_{22}=g_{3} k_{1}+\frac{(1-\mu)}{2 P^{2}} g_{3} k_{2}+\frac{(1-\mu) \alpha^{2}}{2} g_{4} k_{4} \\
r_{33}=\frac{(1-\mu)}{2 P^{2}} g_{3} k_{2}+\frac{(1+\mu)}{2 P^{2}} g_{3} k_{2} \\
k_{1}=\int_{0}^{1} \int_{0}^{1}\left(\frac{d^{2} h}{d R^{2}}\right)^{2} d R d Q \\
k_{2}=\int_{0}^{1} \int_{0}^{1}\left(\frac{d^{2} h}{d R d Q}\right)^{2} d R d Q \\
k_{3}=\int_{0}^{1} \int_{0}^{1}\left(\frac{d^{2} h}{d Q^{2}}\right)^{2} d R d Q \\
k_{4}=\int_{0}^{1} \int_{0}^{1}\left(\frac{d h}{d R}\right)^{2} d R d Q \\
k_{5}=\int_{0}^{1} \int_{0}^{1}\left(\frac{d h}{d Q}\right)^{2} d R d Q \\
F_{r q}=\int_{0}^{1} \int_{0}^{1} h d R d Q
\end{gathered}
$$

\section{SHEARING STRESS DISTRIBUTION IN RECTANGULAR CROSS-SECTIONS}

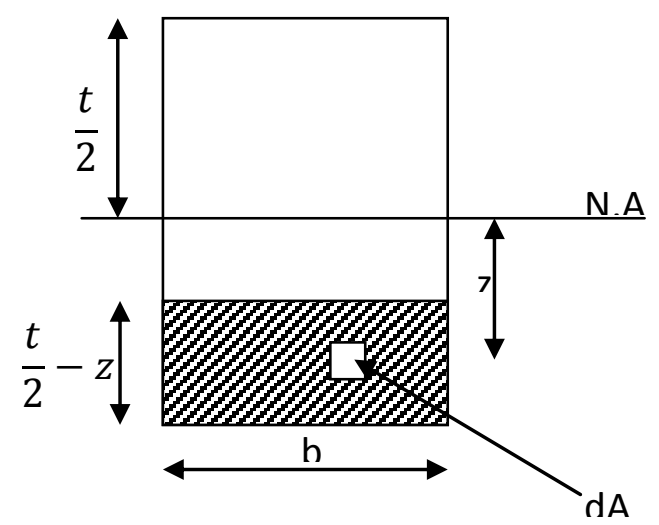

Figure 2: A rectangular cross section

From strength of materials, the equation shear stress is given as:

$$
\tau=\frac{V H}{I b}
$$

Where V, H, I and b are transverse shear force, first moment of area, second moment of inertia and breadth of the section respectively.

Using figure 2 and following mathematically principle the first moment of area is obtained:

$$
H=\int z d A=\frac{b}{2}\left(\frac{t}{2}-z\right)\left(\frac{t}{2}+z\right)
$$




$$
\text { That is, } H=\frac{b}{2}\left(\frac{t^{2}}{4}-z^{2}\right)
$$

The second moment of inertia for a rectangular section is given as:

$$
I=\frac{b t^{2}}{12}
$$

Substituting equations (51) and (52) into equation (50) gives:

$$
\tau=\frac{V}{b t}\left(\frac{3}{2}-6 \frac{z^{2}}{t^{2}}\right)=\frac{V}{b t} G(z)
$$

Where the shear stress profile, $\mathrm{G}(\mathrm{z})$ is:

$$
G(z)=\left(\frac{3}{2}-6 \frac{z^{2}}{t^{2}}\right)
$$

It is assumed here that the shear stress profile, $\mathrm{G}(\mathrm{z})$ is related to shear deformation profile, $\mathrm{F}(\mathrm{z})$ as:

$$
G(z)=\frac{d F(z)}{d z}
$$

Using equations (54) and (55) we obtain:

$$
\begin{aligned}
& F(z)=\frac{3 z}{2}\left(1-\frac{4}{3}\left[\frac{z}{t}\right]^{2}\right) \\
& F(S)=\frac{3 S t}{2}\left(1-\frac{4}{3} S^{2}\right)
\end{aligned}
$$

Where $\mathrm{S}=\mathrm{z} / \mathrm{t}$ (a non-dimensional form of $\mathrm{z}$ )

This function of $\mathrm{z}$ is exactly Krishna Murty Model (KrishnaMurty, 1984) divided by 1.5. However, using the Krishna Murty model will result in unestimating the vertical shear stress by $50 \%$.

Substituting F(S) models into equations (39) to (41) gives g1, g2, g3 and g4 values for different models used herein for numerical examples:

$$
\begin{gathered}
\text { i. } \quad \begin{array}{l}
\text { Present Model } \\
F(S)=\frac{3 S t}{2}\left(1-\frac{4}{3} S^{2}\right)
\end{array} \\
g_{1}=1 ; g_{2}=1.2 ; g_{3}=\frac{51}{35} ; g_{4}=14.4 \\
\text { ii. } \quad \text { Touratier }(1991) \text { model } \\
F(S)=\frac{t}{\pi} \sin (\pi S) \\
g_{1}=1 ; g_{2}=0.774 ; g_{3}=\frac{307}{505} ; g_{4}=6 \\
\text { iii. } \quad \begin{array}{l}
F(S)=S t . \exp \left(2-2 S^{2}\right) \\
g_{1}=1 ; g_{2}=\frac{274}{439} ; g_{3}=\frac{27}{422} ; g_{4}=6.18744
\end{array}
\end{gathered}
$$

\section{DEFINITION OF SOME QUANTITIES}

Recall equation 7:

$$
w=c_{1} h
$$

Let us rewrite it as:

$$
w=c_{1} h=B_{1} h\left(\frac{q a^{4}}{D}\right)=k_{w}\left(\frac{q a^{4}}{D}\right)
$$

This implies that: 


$$
k_{w}=B_{1} \text { hand } B_{1}=\frac{c_{1}}{\left(\frac{q a^{4}}{D}\right)}
$$

Let the displacements of plate under pure bending then be defined as:

$$
\begin{aligned}
& w=\left(\frac{q a^{4}}{D}\right) k_{w} \\
& u=\left(\frac{t q a^{3}}{D}\right) k_{u} \\
& v=\left(\frac{t q a^{3}}{D}\right) k_{v}
\end{aligned}
$$

Where $\mathrm{k}_{\mathrm{w}}$, a and $\mathrm{D}$ are as defined earlier, $\mathrm{q}$ is the uniform distributed load on the plate. $\mathrm{k}_{\mathrm{u}}$ and $\mathrm{k}_{\mathrm{v}}$ are extracted from equations (12) and (13) as:

$$
\begin{gathered}
k_{u}=\left(-B_{1} S+B_{2} F(S)\right) \frac{d h}{d R} \\
k_{v}=\frac{1}{P}\left(-B_{1} S+B_{3} F(S)\right) \frac{d h}{d Q} \\
\text { Where } B_{2}=\frac{c_{2}}{\left(\frac{q a^{4}}{D}\right)} \text { and }_{3}=\frac{c_{3}}{\left(\frac{q a^{4}}{D}\right)}
\end{gathered}
$$

Similarly, let us define the stress components as:

$$
\begin{aligned}
\sigma_{x} & =\frac{E}{1-\mu^{2}}\left(\frac{t q a^{2}}{D}\right) k_{\sigma_{x}} \\
\sigma_{y} & =\frac{E}{1-\mu^{2}}\left(\frac{t q a^{2}}{D}\right) k_{\sigma_{y}} \\
\tau_{x y} & =\frac{E}{1+\mu}\left(\frac{t q a^{2}}{D}\right) k_{\tau_{x y}} \\
\tau_{x z} & =\frac{E}{1+\mu}\left(\frac{q a^{3}}{D}\right) k_{\tau_{x z}} \\
\tau_{y z} & =\frac{E}{1+\mu}\left(\frac{q a^{3}}{D}\right) k_{\tau_{y z}}
\end{aligned}
$$

Where $\mathrm{k}_{\sigma \mathrm{x}}, \mathrm{k}_{\sigma \mathrm{y}}, \mathrm{k}_{\tau \mathrm{\tau x}}, \mathrm{k}_{\tau \mathrm{xz}}$ and $\mathrm{k}_{\tau \mathrm{yz}}$ are defined from equations (24) to (28)as:

$$
\begin{gathered}
k_{\sigma_{x}}=\left[-B_{1} S+B_{2} F(S)\right] \frac{d^{2} h}{d R^{2}}+ \\
\frac{\mu}{P^{2}}\left[-B_{1} S+B_{3} F(S)\right] \frac{d^{2} h}{d Q^{2}} \\
k_{\sigma_{Y}}=\mu\left[-B_{1} S+B_{2} F(S)\right] \frac{d^{2} h}{d R^{2}}+ \\
\frac{1}{P^{2}}\left[-B_{1} S+B_{3} F(S)\right] \frac{d^{2} h}{d Q^{2}} \\
k_{\tau_{x y}}=\frac{1}{2 P}\left[-2 B_{1} S+B_{2} F(S)+B_{3} F(S)\right] \frac{d^{2} h}{d R d Q} \\
k_{\tau_{x z}}=\frac{B_{2}}{2} \frac{d F(S)}{d S} \frac{d h}{d R} \\
k_{\tau_{y z}}=\frac{B_{3}}{2 P} \frac{d F(S)}{d S} \frac{d h}{d Q}
\end{gathered}
$$

Substituting equations (42) and (43) into equations (56) to (58) gives:

$$
\begin{aligned}
& w=12\left(1-\mu^{2}\right) \alpha^{3} k_{w}\left(\frac{q a}{E}\right) \\
& u=12\left(1-\mu^{2}\right) \alpha^{3} k_{u}\left(\frac{t q}{E}\right) \\
& v=12\left(1-\mu^{2}\right) \alpha^{3} k_{v}\left(\frac{t q}{E}\right)
\end{aligned}
$$

Similarly, substituting equations (42) and (43) into equations (61) to (65) gives:

$$
\begin{aligned}
& \sigma_{x}=k_{\sigma_{x}}\left(12 \alpha^{2}\right) q \\
& \sigma_{y}=k_{\sigma_{y}}\left(12 \alpha^{2}\right) q \\
& \tau_{x y}=k_{\tau_{x y}}\left[12 \alpha^{2}(1-\mu)\right] q
\end{aligned}
$$




$$
\begin{aligned}
\tau_{x z} & =k_{\tau_{x z}}\left[12 \alpha^{3}(1-\mu)\right] q \\
\tau_{y z} & =k_{\tau_{y z}}\left[12 \alpha^{3}(1-\mu)\right] q
\end{aligned}
$$

Let us define non-dimensional form of the displacements and stress components according to Sayyad et al.

(2012) as:

$$
\begin{gathered}
\bar{w}=\frac{100 E w}{q t \alpha^{4}}(79) ; \quad \bar{u}=\frac{u E}{q t \alpha^{3}}(80) \\
\bar{v}=\frac{v E}{q t \alpha^{3}}(81) ; \quad \bar{\sigma}_{x}=\frac{\sigma_{x}}{q \alpha^{2}}(82) \\
\bar{\sigma}_{y}=\frac{\sigma_{y}}{q \alpha^{2}}(83) ; \quad \bar{\tau}_{x y}=\frac{\tau_{x y}}{q \alpha^{2}}(84) \\
\bar{\tau}_{x z}=\frac{\tau_{x z}}{q \alpha}(85) ; \quad \bar{\tau}_{y z}=\frac{\tau_{y z}}{q \alpha}(86)
\end{gathered}
$$

Using equations (71) to (78), we define the non-dimensional form of the displacements and stress components as:

$$
\begin{gathered}
\bar{w}=1200\left(1-\mu^{2}\right) k_{w}(87) \\
\bar{u}=12\left(1-\mu^{2}\right) k_{u}(88) \\
\bar{v}=12\left(1-\mu^{2}\right) k_{v}(89) \\
\bar{\sigma}_{x}=12 k_{\sigma_{x}}(90) \\
\bar{\sigma}_{y}=12 k_{\sigma_{y}}(91) \\
\bar{\tau}_{x y}=12(1-\mu) k_{\tau_{x y}}(92) \\
\bar{\tau}_{x z}=12 \alpha^{2}(1-\mu) k_{\tau_{x z}}(93) \\
\bar{\tau}_{y z}=12 \alpha^{2}(1-\mu) k_{\tau_{y z}}(94)
\end{gathered}
$$

\section{NUMERICAL PROBLEM}

Determine the deflection at the center $(0.5,0.5,0)$ of ssss thick plate. Where $(0.5,0.5,0)$ means $\mathrm{R}=0.5 ; \mathrm{Q}=$ $0.5 ; \mathrm{S}=0$. Determine also the in-plane normal stresses at $(0.5,0.5,0.5)$, in-plane shear stress at $(0,0,0.5)$ and the vertical shear stress $\left(\tau_{\mathrm{xz}}\right)$ at $(0,0.5,0)$ of thessss plate. Polynomial and trigonometric displacement shall be used. Function.

The polynomial displacement function, $\mathrm{h}$ is given as:

$h=\left(R-2 R^{3}+R^{4}\right)\left(Q-2 Q^{3}+Q^{4}\right) \quad(57)$

The trigonometric displacement function, $\mathrm{h}$ is given as:

$h=\sin \pi R \sin \pi Q$

The $\mathrm{k}$ values for both polynomial and trigonometric functions are given on table 1 .

Table 1: The values of $\mathrm{k}$ and Frq for polynomial and trigonometric functions

\begin{tabular}{|l|l|l|}
\hline & Polynomial & Trigonometry \\
\hline $\mathrm{k} 1$ & 0.23621 & 24.352273 \\
\hline $\mathrm{k} 2$ & 0.23591 & 24.35227 \\
\hline $\mathrm{k} 3$ & 0.23621 & 24.35227 \\
\hline $\mathrm{k} 4$ & 0.0239 & 2.467401 \\
\hline $\mathrm{k} 5$ & 0.0239 & 2.467401 \\
\hline $\mathrm{F}_{\mathrm{rq}}$ & 0.04 & $92 / 227$ \\
\hline
\end{tabular}

\section{RESULTS AND DISCUSSIONS}

A close look at tables 1 to 8 reveals that the values from the polynomial and trigonometric shape functions of the present study are quite close to each other. The recorded difference is attributed to the fact that the two shape functions are only approximations of each other. Two of them are not exactly the same. Furthermore, it is revealed that the values from trigonometric shape function based on the model of present study agree very well with the values based on Krishna model. However, a critical observation of the values reveals that the values from different models are very good approximation of one another. Table 3, 4, 7 and 8 showed that at spandepth ratio of up to 100 and above, the values obtained from the models used herein coincide exactly with values from CPT. This is quite expected since we assumed in CPT analyses that at span-depth ratios of up to 100, a plate can be taking as being thin. Hence, the model derived herein is quite sufficient for thick plate (refined plate theory) analyses. It shall also be deduced that polynomial shape functions are also adequate for thick plate analyses. Most works on thick plate analyses revolve around use of trigonometric shape function and some other functions. 
Use of Polynomial shape function in Shear Deformation Theory for Thick Plate Analysis

Table 2: Non dimensional parameters for $\mathrm{a} / \mathrm{t}=4$

\begin{tabular}{|l|c|c|c|c|c|r|}
\hline $\mathrm{a} / \mathrm{b}=1$ & $\bar{u}$ & $\bar{w}$ & $\overline{\sigma_{x}}$ & $\overline{\sigma_{y}}$ & $\overline{\tau_{x y}}$ & $\overline{\tau_{x z}}$ \\
\hline Present (P) & -0.0765 & 6.1044 & 0.3279 & 0.3279 & -0.1883 & 0.3906 \\
\hline Present (T) & -0.0755 & 6.1383 & 0.3390 & 0.3390 & -0.1826 & 0.3854 \\
\hline Karama (T) & -0.0769 & 6.1035 & 0.3452 & 0.3452 & -0.1859 & 0.3849 \\
\hline Touratier (T) & -0.0758 & 6.1342 & 0.3402 & 0.3402 & -0.1832 & 0.3973 \\
\hline Krishna (T) & -0.0756 & 6.1483 & 0.3392 & 0.3392 & -0.1826 & 0.3878 \\
\hline Pagano (Exact) & 0.0720 & 5.6940 & 0.3070 & - & - & 0.3067 \\
\hline CPT (P) & -0.0723 & 4.5175 & 0.3098 & 0.3098 & -0.1779 & 0.0000 \\
\hline CPT (T) & -0.0071 & 4.5434 & 0.3203 & 0.3203 & -0.1725 & 0.0000 \\
\hline
\end{tabular}

Legend: $(\mathrm{P})$ means the shape function is polynomial

(T) means the shape function is trigonometry

Table 3: Non dimensional parameters for $\mathrm{a} / \mathrm{t}=10$

\begin{tabular}{|l|r|r|r|r|r|r|}
\hline $\mathrm{a} / \mathrm{b}=1$ & \multicolumn{1}{c|}{$\bar{u}$} & \multicolumn{1}{c|}{$\bar{w}$} & \multicolumn{1}{c|}{$\overline{\sigma_{x}}$} & \multicolumn{1}{c|}{$\overline{\sigma_{y}}$} & \multicolumn{1}{c|}{$\overline{\tau_{x y}}$} & \multicolumn{1}{c|}{$\overline{\tau_{x z}}$} \\
\hline Present (P) & -0.0730 & 4.7723 & 0.3127 & 0.3127 & -0.1796 & 0.3920 \\
\hline Present (T) & -0.0720 & 4.7995 & 0.3233 & 0.3233 & -0.1741 & 0.3868 \\
\hline Karama (T) & -0.0723 & 4.7970 & 0.3243 & 0.3243 & -0.1746 & 0.3909 \\
\hline Touratier (T) & -0.0721 & 4.7991 & 0.3235 & 0.3235 & -0.1742 & 0.3991 \\
\hline Krishna (T) & -0.0720 & 4.8011 & 0.3233 & 0.3233 & -0.1741 & 0.3892 \\
\hline Pagano (Exact) & 0.0730 & 4.6390 & 0.2890 & - & - & 0.3247 \\
\hline CPT (P) & -0.0723 & 4.5175 & 0.30977 & 0.30977 & -0.17792 & 0.0000 \\
\hline CPT (T) & -0.0714 & 4.5434 & 0.3203 & 0.3203 & -0.1725 & 0.0000 \\
\hline
\end{tabular}

Table 4: Non dimensional parameters for $\mathrm{a} / \mathrm{t}=100$

\begin{tabular}{|l|r|r|r|r|r|r|}
\hline $\mathrm{a} / \mathrm{b}=1$ & $\bar{u}$ & $\bar{w}$ & $\overline{\sigma_{x}}$ & $\overline{\sigma_{y}}$ & $\overline{\tau_{x y}}$ & \multicolumn{1}{c|}{$\overline{\tau_{x z}}$} \\
\hline Present (P) & -0.0723 & 4.5201 & 0.3098 & 0.3098 & -0.1779 & 0.3920 \\
\hline Present (T) & -0.0714 & 4.5460 & 0.3203 & 0.3203 & -0.1725 & 0.3868 \\
\hline Karama (T) & -0.0714 & 4.5460 & 0.3203 & 0.3203 & -0.1725 & 0.3909 \\
\hline Touratier (T) & -0.0714 & 4.5460 & 0.3203 & 0.3203 & -0.1725 & 0.3991 \\
\hline Krishna (T) & -0.0714 & 4.5460 & 0.3203 & 0.3203 & -0.1725 & 0.3892 \\
\hline CPT (P) & -0.0723 & 4.5175 & 0.3098 & 0.3098 & -0.1779 & 0.3247 \\
\hline CPT (T) & -0.0714 & 4.5434 & 0.3203 & 0.3203 & -0.1725 & 0.0000 \\
\hline
\end{tabular}

Table 5: Non dimensional parameters for $\mathrm{a} / \mathrm{t}=1000$

\begin{tabular}{|l|r|r|r|r|r|r|}
\hline $\mathrm{a} / \mathrm{b}=1$ & $\bar{u}$ & $\bar{w}$ & $\overline{\sigma_{x}}$ & \multicolumn{1}{c|}{$\overline{\sigma_{y}}$} & $\overline{\tau_{x y}}$ & \multicolumn{1}{c|}{$\overline{\tau_{x z}}$} \\
\hline Present (P) & -0.0723 & 4.5175 & 0.3098 & 0.3098 & -0.1779 & 0.3923 \\
\hline Present (T) & -0.0714 & 4.5435 & 0.3203 & 0.3203 & -0.1725 & 0.3870 \\
\hline Karama (T) & -0.0714 & 4.5435 & 0.3203 & 0.3203 & -0.1725 & 0.3921 \\
\hline Touratier (T) & -0.0714 & 4.5435 & 0.3203 & 0.3203 & -0.1725 & 0.3994 \\
\hline Krishna (T) & -0.0714 & 4.5435 & 0.3203 & 0.3203 & -0.1725 & 0.3895 \\
\hline CPT (P) & -0.0723 & 4.5175 & 0.3098 & 0.3098 & -0.1779 & 0.0000 \\
\hline CPT (T) & -0.0714 & 4.5434 & 0.3203 & 0.3203 & -0.1725 & 0.0000 \\
\hline
\end{tabular}


Use of Polynomial shape function in Shear Deformation Theory for Thick Plate Analysis

Table 6: Dimensional parameters for $\mathrm{a} / \mathrm{t}=4$

\begin{tabular}{|l|c|c|c|c|c|c|}
\hline $\mathrm{a} / \mathrm{b}=1$ & \multicolumn{1}{|c|}{$\mathrm{u}$} & $\mathrm{w}$ & \multicolumn{1}{c|}{$\sigma_{\mathrm{x}}$} & \multicolumn{1}{c|}{$\sigma_{\mathrm{y}}$} & $\tau_{\mathrm{xy}}$ & \multicolumn{1}{c|}{$\tau_{\mathrm{xz}}$} \\
\hline & $\mathrm{tqa}^{3} / \mathrm{D}$ & $\mathrm{qa}^{4} / \mathrm{D}$ & $\mathrm{tqa}^{2} / \mathrm{D}$ & $\mathrm{tqa}^{2} / \mathrm{D}$ & $\mathrm{tqa}^{2} / \mathrm{D}$ & $\mathrm{qa}^{3} / \mathrm{D}$ \\
\hline Present (P) & -0.0070 & 0.00559 & 0.03003 & 0.03003 & -0.0172 & 0.00149 \\
\hline Present (T) & -0.0069 & 0.00562 & 0.03105 & 0.03105 & -0.0167 & 0.00147 \\
\hline Karama (T) & -0.0070 & 0.00559 & 0.03161 & 0.03161 & -0.0170 & 0.00147 \\
\hline Touratier (T) & -0.0069 & 0.00562 & 0.03115 & 0.03115 & -0.0168 & 0.00152 \\
\hline Krishna (T) & -0.0069 & 0.00563 & 0.03106 & 0.03106 & -0.0167 & 0.00148 \\
\hline & & & & & & \\
Pagano (Exact) & 0.0066 & 0.00521 & 0.02811 & - & - & 0.00117 \\
\hline CPT (P) & -0.0066 & 0.00414 & 0.02837 & 0.02837 & -0.0163 & 0.00000 \\
\hline CPT (T) & -0.0065 & 0.00416 & 0.02933 & 0.02933 & -0.0158 & 0.00000 \\
\hline
\end{tabular}

Table 7: Dimensional parameters for $\mathrm{a} / \mathrm{t}=10$

\begin{tabular}{|l|c|c|c|c|c|c|}
\hline $\mathrm{a} / \mathrm{b}=1$ & $\mathrm{u}$ & $\mathrm{w}$ & $\sigma_{\mathrm{x}}$ & $\sigma_{\mathrm{y}}$ & $\tau_{\mathrm{xy}}$ & $\tau_{\mathrm{xz}}$ \\
\hline & $\mathrm{tqa}^{3} / \mathrm{D}$ & $\mathrm{qa}^{4} / \mathrm{D}$ & $\mathrm{tqa}^{2} / \mathrm{D}$ & $\mathrm{tqa}^{2} / \mathrm{D}$ & $\mathrm{tqa}^{2} / \mathrm{D}$ & $\mathrm{qa}^{3} / \mathrm{D}$ \\
\hline Present (P) & -0.0067 & 0.00437 & 0.02863 & 0.02863 & -0.0164 & 0.00024 \\
\hline Present (T) & -0.0066 & 0.00440 & 0.02961 & 0.02961 & -0.0159 & 0.00024 \\
\hline Karama (T) & -0.0066 & 0.00439 & 0.02970 & 0.02970 & -0.0160 & 0.00024 \\
\hline & & & & & & \\
Touratier (T) & -0.0066 & 0.00439 & 0.02962 & 0.02962 & -0.0160 & 0.00024 \\
\hline Krishna (T) & -0.0066 & 0.00440 & 0.02961 & 0.02961 & -0.0159 & 0.00024 \\
\hline Pagano (Exact) & 0.0067 & 0.00425 & 0.02647 & - & - & 0.00020 \\
\hline CPT (P) & -0.0066 & 0.00414 & 0.02837 & 0.02837 & -0.0163 & 0.00000 \\
\hline CPT (T) & -0.0065 & 0.00416 & 0.02933 & 0.02933 & -0.0158 & 0.00000 \\
\hline
\end{tabular}

Table8:Dimensional parameters for $\mathrm{a} / \mathrm{t}=100$

\begin{tabular}{|l|c|c|c|c|c|c|}
\hline $\mathrm{a} / \mathrm{b}=1$ & $\mathrm{u}$ & $\mathrm{w}$ & $\sigma_{\mathrm{x}}$ & $\sigma_{\mathrm{y}}$ & $\tau_{\mathrm{xy}}$ & $\tau_{\mathrm{xz}}$ \\
\hline & $\mathrm{tqa}^{3} / \mathrm{D}$ & $\mathrm{qa}^{4} / \mathrm{D}$ & $\mathrm{tqa}^{2} / \mathrm{D}$ & $\mathrm{tqa}^{2} / \mathrm{D}$ & $\mathrm{tqa}^{2} / \mathrm{D}$ & $\mathrm{qa}^{3} / \mathrm{D}$ \\
\hline Present (P) & -0.0066 & 0.00414 & 0.02837 & 0.02837 & -0.0163 & 0.00000 \\
\hline Present (T) & -0.0065 & 0.00416 & 0.02933 & 0.02933 & -0.0158 & 0.00000 \\
\hline Karama (T) & -0.0065 & 0.00416 & 0.02934 & 0.02934 & -0.0158 & 0.00000 \\
\hline Touratier (T) & -0.0065 & 0.00416 & 0.02933 & 0.02933 & -0.0158 & 0.00000 \\
\hline Krishna (T) & -0.0065 & 0.00416 & 0.02933 & 0.02933 & -0.0158 & 0.00000 \\
\hline CPT (P) & -0.0066 & 0.00414 & 0.02837 & 0.02837 & -0.0163 & 0.00000 \\
\hline CPT (T) & -0.0065 & 0.00416 & 0.02933 & 0.02933 & -0.0158 & 0.00000 \\
\hline
\end{tabular}

Table9:Dimensional parameters for $\mathrm{a} / \mathrm{t}=1000$

\begin{tabular}{|l|c|c|c|c|c|c|}
\hline $\mathrm{a} / \mathrm{b}=1$ & $\mathrm{u}$ & $\mathrm{w}$ & $\sigma_{\mathrm{x}}$ & $\sigma_{\mathrm{y}}$ & $\tau_{\mathrm{xy}}$ & $\tau_{\mathrm{xz}}$ \\
\hline & $\mathrm{tqa}^{3} / \mathrm{D}$ & $\mathrm{qa}^{4} / \mathrm{D}$ & $\mathrm{tqa}^{2} / \mathrm{D}$ & $\mathrm{tqa}^{2} / \mathrm{D}$ & $\mathrm{tqa}^{2} / \mathrm{D}$ & $\mathrm{qa}^{3} / \mathrm{D}$ \\
\hline Present (P) & -0.0066 & 0.00414 & 0.02837 & 0.02837 & -0.0163 & 0.00000 \\
\hline Present (T) & -0.0065 & 0.00416 & 0.02933 & 0.02933 & -0.0158 & 0.00000 \\
\hline Karama (T) & -0.0065 & 0.00416 & 0.02933 & 0.02933 & -0.0158 & 0.00000 \\
\hline Touratier (T) & -0.0065 & 0.00416 & 0.02933 & 0.02933 & -0.0158 & 0.00000 \\
\hline Krishna (T) & -0.0065 & 0.00416 & 0.02933 & 0.02933 & -0.0158 & 0.00000 \\
\hline CPT (P) & -0.0066 & 0.00414 & 0.02837 & 0.02837 & -0.0163 & 0.00000 \\
\hline CPT (T) & -0.0065 & 0.00416 & 0.02933 & 0.02933 & -0.0158 & 0.00000 \\
\hline
\end{tabular}




\section{REFERENCES}

[1] S.A. Sadrnejad, A. SaediDaryan and M. Ziaei (2009). Vibration Equations of Thick Rectangular Plates Using Mindlin Plate Theory. Journal of Computer Science 5 (11): 838-842, 2009 ISSN 1549-3636

[2] T. H. Daouadji, A. Tounsi and El A. A. Bedia (2013).A New Higher Order Shear Deformation Model for Static Behavior of Functionally Graded Plates. Advances in Applied Mathematics and Mechanics Adv. Appl. Math. Mech., Vol. 5, No. 3, pp. 351-364

[3] S. Sayyada Y. M. Ghugal (2012). Bending and free vibration analysis of thick isotropic plates by using exponential shear deformation theory. Applied and Computational Mechanics 6, pp. 65-82

[4] M. V.V. Murthy (1981). An Improved Transverse Shear Deformation Theory for Laminated Anisotropic Plates. NASA Technical Paper 1903

[5] A. S. Sayyad (2011). Comparison of various shear deformation theories for the free vibration of thick isotropic beams. INTERNATIONAL JOURNAL OF CIVIL AND STRUCTURAL ENGINEERING Volume 2, No 1,pp. 85-97

[6] S.H. Hashemi, M. Arsanjani (2005). Exat characteristic equations for some of classical boundary conditions of vibrating moderately thick rectangular plate. International Journal of Solids and Structures 42 (2005) 819-853

[7] S.B.Chikalthankar, I.I.Sayyad, V.M.Nandedkar (2013).Analysis of Orthotropic Plate By Refined Plate Theory. International Journal of Engineering and Advanced Technology (IJEAT) ISSN: 2249 - 8958, Volume-2, Issue-6, pp. 310-315

[8] B. Sidda Reddy (2014).Bending Behaviour Of Exponentially Graded Material Plates Using New Higher Order Shear Deformation Theory with Stretching Effect . International Journal of Engineering Research ISSN:2319-6890)(online),2347-5013(print) Volume No.3 Issue No: Special 1, pp: 124-131

[9] M. K. Matikainen, A. L. Schwab and Aki M. Mikkola (2009). Comparison of two moderately thick plate elements based on the absolute nodal coordinate formulation. MULTIBODY DYNAMICS 2009, ECCOMAS Thematic Conference K. Arczewski, J. Fra czek, M. Wojtyra (eds.) Warsaw, Poland, 29 June-2 July 2009.

[10] T. H.Daouadji, A. Tounsi, L. Hadji, A. H. Henni, A. B. El Abbes (2012). A theoretical analysis for static and dynamic behavior of functionally graded plates. Materials Physics and Mechanics 14 (2012) 110-128

[11] C. Zhen-qiang, W.Xiu:xiand H. Mao-guang (1994). Postbuckling behavior of rectangular moderately thick plates and sandwich plates. Applied Mathematics and Mechanics (English Edition, Vol. 15, No. 7, July 1994).

[12] R.P. Shimpi, H. G. Patel (2006).A two variable refined plate theory for orthotropic plate analysis. International Journal of Solids and Structures 43 (2006) 6783-6799

[13] S. Goswami, W. Becker (2013). A New Rectangular Finite Element Formulation Based on Higher Order Displacement Theory for Thick and Thin Composite and Sandwich Plates. World Journal of Mechanics, 2013, 3, 194-201

[14] M. A. V. Krishna (1984), Toward a consistent beam theory, AIAA Journal, 22, pp 811-816.

[15] S. A. Ambartsumian (1958), On the theory of bending plates, Izvotd Tech Nauk an Sssr, 5, pp 69-77.

[16] E. T. Kruszewski (1949), Effect of transverse shear and rotatory inertia on the natural frequency of a uniform beam, NACA TN, 1909.

[17] M. Karama, K. S. Afaq and S. Mistou (2003), Mechanical behavior of laminated composite beam by new multi-layered laminated composite structures model with transverse shear stress continuity, International Journal of Solids and Structures, 40, pp 1525-46.

[18] M. Touratier (1991), An efficient standard plate theory, International Journal of Engineering Science, 29(8), pp 901-16.

[19] S. P. Timoshenko, And S. Woinowsky-krieger (1970). Theory of plates and shells (2nd Ed.). Singapore: Mc Graw-Hill Book Co. P.379.

[20] Pagano, N. J., Exact solutions for bidirectional composites and sandwich plates, Journal of Composite Materials 4 (1970) 20-34. 